\title{
Adesão aos acordos voluntários de redução de sódio no Brasil
}

\author{
Adherence to voluntary sodium reduction agreements in Brazil
}

Camila Zancheta Ricardo (https://orcid.org/0000-0003-3643-302X) ${ }^{1}$

Giovanna Calixto Andrade (https://orcid.org/0000-0002-1901-3093) ${ }^{1}$

Bianka Caliman Salvador (https://orcid.org/0000-0003-0912-1901) ${ }^{2}$

Laís Amaral Mais (https://orcid.org/0000-0003-0993-2798) ${ }^{3}$

Ana Clara Duran (https://orcid.org/0000-0001-7317-5790) ${ }^{1}$

Ana Paula Bortoletto Martins (https://orcid.org/0000-0003-3560-4472) ${ }^{3}$
${ }^{1}$ Núcleo de Pesquisas Epidemiológicas em Nutrição e Saúde,

Universidade de São Paulo. Av. Dr. Arnaldo 715. 0124690 São Paulo SP Brasil. ca.zancheta@gmail.com ${ }^{2}$ Secretaria Municipal de Saúde, Prefeitura da Cidade de São Paulo. São Paulo SP Brasil.

${ }^{3}$ Instituto Brasileiro de

Defesa do Consumidor. São Paulo SP Brasil.

\begin{abstract}
The objective was to assess adherence to voluntary agreements for sodium reduction firmed between the food industries and the Ministry of Health in Brazil and to compare their targets with the limit proposed in the Pan American Health Organization (PAHO) nutritional profile model. We used data from 1.553 foods from $32 \mathrm{ca}$ tegories included in the agreements and sold in the largest Brazilian supermarket chains in 2017. The frequency of products with sodium equal or below the cut-offs proposed by the voluntary agreements and by PAHO was calculated. Classification concordance according to the two was evaluated with Cohen's kappa coefficient ( $k$ ). Our results showed that $77.7 \%$ of products were adequate according to the voluntary agreements, and only $35.9 \%$ of them, according to the PAHO model. We identified a weak degree of concordance between both criteria in classifying a product as adequate about sodium content $(k=0.199)$. In conclusion, the voluntary agreements for sodium reduction are limited in their scope and rigor. The adoption of measures oriented for all products, with more restrictive and mandatory targets, should be considered in the country.
\end{abstract}

Key words Sodium chloride, Dietary, Food labeling, Nutrition programs and policies
Resumo O objetivo do trabalho foi avaliar a adesão aos acordos voluntários de redução de sódio firmados entre indústrias de alimentos e o Ministério da Saúde e comparar as metas adotadas com o limite de sódio proposto no modelo de perfil nutricional da Organização Pan-Americana da Saúde (OPAS). Utilizaram-se informações de 1.553 alimentos de 32 categorias incluídas nos acordos e comercializados nas maiores redes de supermercados brasileiras em 2017. Foram calculadas as proporções de produtos com quantidade de sódio igual ou abaixo do limite proposto pelos acordos e pela OPAS. A concordância de classificação dos itens segundo os dois critérios foi avaliada com o coeficiente kappa de Cohen ( $k$ ). Nossos resultados mostraram que $77,7 \%$ dos alimentos analisados estavam adequados segundo os acordos de redução de sódio, porém apenas 35,9\%, segundo o modelo da OPAS. A concordância entre os dois critérios ao classificar um produto como adequado em relação ao conteúdo de sódio foi fraca $(k=0,199)$. Conclui-se que os acordos voluntários de redução de sódio são limitados em relação à abrangência e ao rigor das metas estabelecidas. A adoção de medidas voltadas a todos os produtos disponiveis, com metas mais restritivas e obrigatórias, deveria ser considerada no país.

Palavras-chave Cloreto de sódio na dieta, Rotulagem de alimentos, Programas e politicas de nutrição e alimentação 


\section{Introdução}

As doenças crônicas não transmissíveis (DCNT) são as principais causas de mortalidade em todo o mundo, responsáveis por mais de 40 milhões de óbitos em 2016, o equivalente a $71 \%$ do total ${ }^{1}$. As DCNT que mais contribuem para a carga de morbimortalidade têm fatores de risco comportamentais modificáveis relacionados ao estilo de vida, incluindo consumo abusivo de álcool, tabagismo, inatividade física e alimentação não saudável ${ }^{1}$.

Entre os riscos relacionados à alimentação, destaca-se o consumo excessivo de sódio, que está associado ao aumento da pressão arterial e ao desenvolvimento de doenças cardiovasculares $(\mathrm{DCV})^{2,3}$. Globalmente, o consumo médio de sódio é de 10 gramas por dia, o dobro do recomendado pela Organização Mundial da Saúde (OMS), e a adoção de ações governamentais para redução do consumo populacional é uma medida custo efetiva para prevenção de DCV e mortes prematuras ${ }^{4}$. A OMS incluiu a redução do consumo de sódio como prioridade na prevenção e controle das DCNT, estabelecendo como meta uma redução de $30 \%$ no consumo médio populacional até o ano de $2025^{5}$. A Organização Pan-Americana da Saúde (OPAS), no mesmo sentido, reconheceu o tema como prioritário e lançou uma série de recomendações para que o consumo diário na região fosse menor do que dois gramas de sódio por pessoa, o equivalente a cinco gramas de sal, até $2020^{6}$.

O Brasil está bastante próximo do cenário mundial no que se refere à prevalência e mortalidade por DCNT e ao elevado consumo de sódio. Em 2016, estimou-se que 74\% do total de óbitos foram decorrentes de DCNT, dos quais $28 \%$ causados por $\mathrm{DCV}^{1}$, e o consumo de sódio era aproximadamente o dobro do limite estabelecido pela OMS ${ }^{1,7,8}$. Embora a maior parte do sódio disponível para consumo no país ainda fosse proveniente do sal de cozinha e de condimentos à base de sal, houve um aumento significativo na contribuição de alimentos processados e ultraprocessados para a disponibilidade de sódio no país quando comparados os resultados de compras de alimentos registrados nas Pesquisas de Orçamentos Familiares (POF) realizadas em 2003 e $2009^{8}$. Um estudo que utilizou os dados de consumo também coletados na POF, que incluiu mais de 34 mil brasileiros maiores de dez anos de idade, mostrou que produtos processados e ultraprocessados já contribuíam com mais da metade do sódio dietético consumido pela população brasileira em $2009^{9}$.
Considerando esse panorama, desde 2010 o Ministério da Saúde (MS) promove discussões relacionadas à redução do consumo de sódio. Os eixos prioritários de ação definidos no país foram a promoção de alimentos básicos minimamente processados, a educação alimentar, a orientação sobre o uso de rotulagem nutricional e a reformulação de alimentos processados ${ }^{10}$. Em 2011, o MS e a Associação Brasileira da Indústria de Alimentos (ABIA) assinaram o primeiro acordo voluntário de redução do conteúdo de sódio em alimentos embalados comercializados no Brasil. Entre 2011 e 2017, foram assinados cinco acordos com metas de redução de sódio para 35 categorias de alimentos $^{11-15}$. Vale mencionar que todos os acordos visam compactuar com as estratégias de redução do consumo de sal pela população brasileira para menos de cinco gramas diários até 2020, alinhados com as metas estipuladas pela OPAS e pela OMS ${ }^{11-15}$.

Com o intuito de prover mais evidências da presença de sódio em alimentos embalados disponíveis no mercado brasileiro e contribuir para as políticas públicas implementadas em nível nacional com vistas à redução do consumo de sódio pela população brasileira, este artigo teve como objetivo avaliar a adequação de produtos embalados comercializados no Brasil em relação aos acordos voluntários de redução de sódio e comparar a quantidade de sódio presente nestes alimentos com a recomendação da Organização Pan-Americana da Saúde (OPAS) para a prevenção de DCNT.

\section{Métodos}

As informações do conteúdo de sódio foram coletadas a partir do registro fotográfico de rótulos de alimentos embalados comercializados nas cinco maiores redes brasileiras de supermercados ${ }^{16}$. Nossa coleta ocorreu entre os meses de abril e julho de 2017 em São Paulo, escolhida por ser a maior cidade do país. Como uma das redes de supermercados tinha todas as suas lojas na região Nordeste, para essa rede a coleta foi realizada em Salvador, a maior cidade da região. Todos os estabelecimentos das cinco redes foram georrefenciados e o nível socioeconômico da vizinhança determinado a partir da renda média do chefe de família dos setores censitários localizados em um raio de 1.000 metros a partir da localização das lojas. Utilizamos os dados de renda disponibilizados pelo último censo demográfico brasileiro, realizado em $2010^{17}$. As lojas foram divididas em tercis de renda, e aquelas com maior área física pertencentes ao primeiro e ter- 
ceiro tercis foram incluídas em nossa amostra. Por fim, foram visitadas dois estabelecimentos de cada rede, exceto para uma delas, que autorizou a coleta apenas em um centro de distribuição em que todos os seus produtos estavam disponíveis. Este estudo seguiu a abordagem proposta pelo International Network for Food and Obesity/Non-Communicable Diseases Research, Monitoring and Action Support (INFORMAS), descrita em detalhes por Kanter e colaboradores ${ }^{18}$. Obtivemos a permissão formal das redes de supermercados para a coleta de dados.

Os alimentos e bebidas embalados disponíveis nas lojas visitadas tiveram todos os lados da embalagem fotografados. A digitação dos dados foi feita na plataforma on-line RedCap, em formulário desenvolvido em parceria com a University of North Carolina at Chapel Hill, nos Estados Unidos, e o Instituto de Nutrición y Tecnología de los Alimentos, no Chile, adaptado para o estudo brasileiro. Depois de excluídos produtos duplicados, produtos disponíveis em mais de um tamanho de embalagem, embalagens com múltiplos itens distintos e produtos sem informação, 11.434 produtos foram mantidos no banco de dados.

Para esta análise, foram considerados apenas os produtos pertencentes às 35 categorias de alimentos incluídos nos termos de cooperação para a redução de sódio no Brasil assinados anteriormente à coleta de dados (Quadro 1). Não consideramos, portanto, o acordo firmado em 2017. Esse último acordo estabeleceu novas metas para macarrão instantâneo, pão de forma e bisnaguinha, vigentes para os anos de 2018 e $2020^{15}$. Não encontramos itens correspondentes às categorias "pão francês", "rocambole" e "caldo líquido/gel" em nosso banco de dados. Ao final, 1.553 itens de 32 categorias de alimentos foram incluídos no estudo.

Foram descritos a média e o desvio padrão, bem como os percentis 25,50 e 75 e os valores mínimo e máximo do conteúdo de sódio em cada categoria de alimentos. O teste de Shapiro Wilk foi aplicado para avaliar a normalidade da distribuição do conteúdo de sódio nas categorias de alimentos, considerando como significativos valores de $\mathrm{p} \leq 0,05$. O conteúdo de sódio não apresentou distribuição normal para a maioria das categorias (20 de 32, ou 62,5\%), porém, como as metas de redução consideraram inicialmente o conteúdo médio de sódio das categorias, apresentamos os resultados considerando tanto a média quanto a mediana do conteúdo de sódio dos alimentos.

Para avaliar a adesão aos acordos voluntários, foi calculada a prevalência de produtos com conteúdo de sódio abaixo da meta mais recente es- tabelecida para a categoria. Como incluímos no estudo todos os produtos coletados, analisamos se as marcas que tinham ao menos cinco produtos com conteúdo acima das metas pertenciam a empresas que haviam firmado o acordo.

Adicionalmente, foi verificada a adequação do conteúdo de sódio segundo o Modelo de Perfil Nutricional da OPAS, que estipula como limite para sódio 1 miligrama por quilocaloria do produto $^{19}$. Esse instrumento foi desenvolvido para ser utilizado pelos estados membros da OPAS na identificação de alimentos e bebidas não alcoólicas com perfil nutricional inadequado e sujeitos a medidas regulatórias com o objetivo de prevenir e controlar DCNT relacionadas à alimentação ${ }^{19}$.

Escolhemos esse critério para avaliar o rigor do acordo voluntário brasileiro por ser coerente com a discussão de políticas na região e porque, até onde sabemos, não existem pontos de corte desenvolvidos especificamente para reformulação de produtos com sódio publicados pela OPAS ou pela OMS.

A concordância de classificação dos alimentos segundo os pontos de corte dos acordos voluntários e da OPAS foi verificada utilizando o coeficiente de kappa de Cohen $(\mathrm{k})$. Os seguintes valores e classificações foram adotados para avaliar a concordância entre os métodos: pobre se $\mathrm{k}$ $<0,00$, fraca se $0,00 \leq \mathrm{k} \leq 0,20$, razoável se $0,21 \leq$ $\mathrm{k} \leq 0,40$, moderada se $0,41 \leq \mathrm{k} \leq 0,60$, substancial se $0,61 \leq \mathrm{k} \leq 0,80$, e quase perfeita se $\mathrm{k}>0,80^{20}$.

As análises foram realizadas utilizando o software Stata v.16.0.

\section{Resultados}

Na Tabela 1, está disponível a descrição do conteúdo de sódio segundo categoria de alimentos. As categorias com maiores conteúdos de sódio foram condimentos ["tempero para arroz" (média 20.075,7; mediana 19.730,0 mg/100g), "caldo em pó/cubo" (média 20.029,7; mediana 20.505,3 mg/100g), "tempero em pasta" (média 19.596,2; mediana $26.840,0 \mathrm{mg} / 100 \mathrm{~g}$ ) e "demais temperos" (média 13.807,7; mediana 14.780,0 mg/100g)] e produtos cárneos ["mortadela conservada em refrigeração" (média 1.443,5; mediana 1.320 .0 $\mathrm{mg} / 100 \mathrm{~g}$ ), "mortadela conservada em temperatura ambiente" (média 1.417,9; mediana 1.350.0 mg/100g), "presuntaria” (média 1.329,9; mediana $1.160 .0 \mathrm{mg} / 100 \mathrm{~g}$ ), "linguiça cozida conservada em temperatura ambiente" (média 1.193,6; mediana $1.187 .0 \mathrm{mg} / 100 \mathrm{~g}$ ) e "linguiça cozida conservada em refrigeração” (média 1.136,6; mediana 1.320.0 
Quadro 1. Resumo dos acordos de cooperação voluntários entre o Ministério da Saúde e a indústria de alimentos para a redução do sódio em alimentos no Brasil.

\begin{tabular}{|c|c|c|c|c|c|c|c|}
\hline \multirow{2}{*}{ Acordo/ data } & \multirow{2}{*}{ Categoria de alimentos } & \multicolumn{6}{|c|}{ Meta (mg/100g) a/Ano } \\
\hline & & 2012 & 2013 & 2014 & 2015 & 2016 & 2017 \\
\hline \multirow{3}{*}{$\begin{array}{l}\text { Termo no } 04 / 2011 \\
/ 07-04-2011\end{array}$} & Macarrão instantâneo & $1.920,7$ & & & & & \\
\hline & Pão de forma & 645 & & 522 & & & \\
\hline & Bisnaguinha & 531 & & 430 & & & \\
\hline \multirow{12}{*}{$\begin{array}{l}\text { Termo no } \\
35 / 2011 / 13-12-2011\end{array}$} & Bolo pronto sem recheio & 392 & & 332 & & & \\
\hline & Bolo pronto recheado & 282 & & 242 & & & \\
\hline & Mistura para bolo aerado & 476 & & 398 & & 334 & \\
\hline & Mistura para bolo cremoso & 349 & & 295 & & 250 & \\
\hline & Rocambole & 221 & & 204 & & & \\
\hline & Pão francês & 616 & & 586 & & & \\
\hline & Salgadinho de milho & 1.090 & & 852 & & 747 & \\
\hline & Batata frita/palha & 650 & & 586 & & 529 & \\
\hline & Maionese & 1.283 & & 1.051 & & & \\
\hline & Biscoito doce & 419 & & 359 & & & \\
\hline & Biscoito salgado ${ }^{b}$ & 923 & & 699 & & & \\
\hline & Biscoito recheado & 389 & & 265 & & & \\
\hline \multirow[t]{7}{*}{ Termo/28-08-2012 } & Cereal matinal & & 579 & & 418 & & \\
\hline & Margarina & & 1.089 & & 715 & & \\
\hline & Caldo em pó/cubo ${ }^{c}$ & & 1.100 & & 1.025 & & \\
\hline & Caldo líquido/gel & & 928 & & 865 & & \\
\hline & Tempero em pasta & & 37.901 & & 33.134 & & \\
\hline & Tempero para arroz & & 32.927 & & 32.076 & & \\
\hline & Demais temperos & & 23.775 & & 21.775 & & \\
\hline \multirow[t]{13}{*}{ Termo/05-11-2013 } & Queijo muçarela & & & 559 & & 512 & \\
\hline & Requeijão & & & 587 & & 541 & \\
\hline & Sopa ${ }^{\mathrm{d}}$ & & & & 327 & & 314 \\
\hline & Sopa individual/instantânea ${ }^{\mathrm{e}}$ & & & & 334 & & 330 \\
\hline & Empanado $^{\mathrm{f}}$ & & & & 690 & & 650 \\
\hline & Hambúrguer ${ }^{\mathrm{g}}$ & & & & 780 & & 740 \\
\hline & $\begin{array}{l}\text { Linguiça cozida conservada em } \\
\text { temperatura ambiente }\end{array}$ & & & & 1.560 & & 1.500 \\
\hline & $\begin{array}{l}\text { Linguiça cozida conservada em } \\
\text { refrigeração }\end{array}$ & & & & 1.310 & & 1.210 \\
\hline & Linguiça frescal & & & & 1.080 & & 970 \\
\hline & Mortadela conservada em refrigeração & & & & 1.270 & & 1.180 \\
\hline & $\begin{array}{l}\text { Mortadela conservada em } \\
\text { temperatura ambiente }\end{array}$ & & & & 1.380 & & 1.350 \\
\hline & Salsicha ${ }^{\mathrm{h}}$ & & & & 1.140 & & 1.120 \\
\hline & Presuntaria ${ }^{\mathrm{i}}$ & & & & 1.180 & & 1.160 \\
\hline
\end{tabular}

${ }^{a}$ Meta expressa em $100 \mathrm{~g}$ de produto conforme disponível para venda, exceto para caldo em pó/cubo, sopas. Ver notas abaixo. ${ }^{\mathrm{b}}$ Tipo cream cracker, água e sal, água. ${ }^{\mathrm{c}}$ Ponto de corte expresso em mg/porção. ${ }^{\mathrm{d}}$ Sopas de caldo translúcido ou cremosas, feitas à base de carnes, aves, vegetais, grãos, entre outros ingredientes, sendo que, quando não oferecidas prontas para o consumo, necessitam de cozimento, sendo excluídos dessa categoria os cremes de cebola que têm indicação exclusiva para uso culinário. Ponto de corte expresso em $\mathrm{mg} / 100 \mathrm{~mL}$ do produto pronto para consumo. ${ }^{e}$ Sopas preparadas apenas por meio da adição de água quente e homogeneização, sem necessidade de cozimento. Ponto de corte expresso em $\mathrm{mg} / 100 \mathrm{~mL}$ do produto pronto para consumo. ${ }^{\mathrm{f}}$ Excluem-se empanados de peixe e empanados cuja matéria-prima principal é vegetal. " Excluem-se hambúrgueres cuja matériaprima principal é vegetal. ${ }^{\text {h }}$ Excluem-se salsichas em conserva (embaladas em vidros ou latas). ${ }^{\mathrm{i}}$ Incluem-se presunto (cozido e de aves), apresuntado e fiambre.

Fonte: Brasil, 2011 $1^{11}, 2011^{12}, 2012^{13}, 2013^{14}$. 
Tabela 1. Número de alimentos e distribuição do conteúdo de sódio em miligramas por 100 gramas do produto, segundo categoria de alimentos.

\begin{tabular}{|c|c|c|c|c|c|c|c|c|}
\hline \multirow{2}{*}{ Categorias de alimentos } & \multirow{2}{*}{$\mathbf{n}$} & \multicolumn{7}{|c|}{ Sódio (mg) em $100 \mathrm{~g}$ de alimento } \\
\hline & & Média & DP & Min & p25 & p50 & p75 & Máx. \\
\hline Macarrão instantâneo & 80 & $1.626,9$ & 281,6 & 843,8 & $1.504,7$ & $1.730,0$ & $1.828,6$ & $1.960,0$ \\
\hline Pão de forma & 112 & 366,9 & 98,5 & 146,0 & 300,0 & 370,0 & 436,0 & 598,1 \\
\hline Bisnaguinha & 9 & 335,3 & 38,9 & 262,0 & 316,0 & 358,0 & 364,0 & 366,0 \\
\hline Bolo pronto sem recheio & 90 & 272,1 & 144,1 & 35,7 & 176,7 & 259,2 & 323,3 & 713,3 \\
\hline Bolo pronto recheado & 46 & 194,2 & 82,5 & 0,0 & 155,0 & 195,8 & 220,0 & 470,0 \\
\hline Mistura para bolo aerado & 78 & 433,1 & 190,7 & 14,3 & 329,7 & 427,6 & 502,7 & 967,6 \\
\hline Mistura para bolo cremoso & 8 & 269,2 & 88,1 & 110,3 & 200,1 & 314,1 & 336,7 & 341,2 \\
\hline Salgadinho de milho & 48 & 752,6 & 252,2 & 50,0 & 620,0 & 720,0 & 884,0 & $1.196,0$ \\
\hline Batata frita/palha & 81 & 434,6 & 151,0 & 22,8 & 352,0 & 476,0 & 524,0 & 748,0 \\
\hline Maionese & 21 & 842,1 & 156,6 & 541,7 & 758,3 & 833,3 & 958,3 & $1.066,7$ \\
\hline Biscoito doce & 37 & 304,6 & 91,9 & 1,3 & 286,7 & 326,7 & 356,7 & 476,7 \\
\hline Biscoito salgado & 45 & 724,2 & 298,7 & 1,2 & 600,0 & 696,7 & 700,0 & $1.613,3$ \\
\hline Biscoito recheado & 103 & 228,5 & 76,4 & 0,0 & 190,0 & 233,3 & 260,0 & 600,0 \\
\hline Cereal matinal & 157 & 138,7 & 147,6 & 0,0 & 15,0 & 112,5 & 223,3 & 761,9 \\
\hline Margarina & 14 & 650,7 & 146,2 & 400,0 & 600,0 & 620,0 & 710,0 & $1.050,0$ \\
\hline Caldo em pó/cubo a & 38 & $20.029,7$ & $4.800,3$ & 40,6 & $18.419,1$ & $20.505,3$ & $23.157,9$ & $27.253,3$ \\
\hline Tempero em pasta & 31 & $19.596,2$ & $14.064,1$ & 0,0 & $3.700,0$ & $26.840,0$ & $32.540,0$ & $35.380,0$ \\
\hline Tempero para arroz & 14 & $20.075,7$ & $3.825,9$ & $12.220,0$ & $19.540,0$ & $19.730,0$ & $20.340,0$ & $31.260,0$ \\
\hline Demais temperos & 66 & $13.807,7$ & $8.242,0$ & 360,0 & $7.457,6$ & $14.780,0$ & $20.220,0$ & $36.740,0$ \\
\hline Queijo muçarela & 53 & 422,7 & 162,9 & 33,3 & 370,0 & 450,0 & 546,7 & 796,7 \\
\hline Requeijão & 85 & 570,0 & 149,7 & 183,3 & 483,3 & 532,0 & 626,7 & $1.100,0$ \\
\hline Sopa $^{2}$ & 43 & 289,1 & 300,8 & 89,0 & 216,0 & 269,2 & 290,4 & $2.111,6$ \\
\hline Sopa individual/instantânea ${ }^{\mathrm{b}}$ & 12 & 312,5 & 47,5 & 245,0 & 282,5 & 311,7 & 339,6 & 411,2 \\
\hline Empanado & 43 & 521,1 & 96,6 & 345,4 & 453,8 & 531,0 & 607,7 & 677,7 \\
\hline Hambúrguer & 39 & 495,0 & 312,2 & 38,0 & 96,2 & 570,5 & 737,5 & $1.211,7$ \\
\hline $\begin{array}{l}\text { Linguiça cozida conservada } \\
\text { em temperatura ambiente }\end{array}$ & 22 & $1.193,6$ & 371,0 & 1,5 & $1.000,0$ & $1.187,0$ & $1.500,0$ & $1.640,0$ \\
\hline $\begin{array}{l}\text { Linguiça cozida conservada } \\
\text { em refrigeração }\end{array}$ & 13 & $1.136,6$ & 237,5 & 780,0 & $1.000,0$ & $1.066,0$ & $1.342,0$ & $1.500,0$ \\
\hline Linguiça frescal & 60 & 958,5 & 213,9 & 434,0 & 852,0 & $1.028,0$ & $1.077,0$ & $1.428,0$ \\
\hline $\begin{array}{l}\text { Mortadela conservada em } \\
\text { refrigeração }\end{array}$ & 21 & $1.443,5$ & 884,2 & 160,0 & $1.232,5$ & $1.320,0$ & $1.415,0$ & $5.000,0$ \\
\hline $\begin{array}{l}\text { Mortadela conservada em } \\
\text { temperatura ambiente }\end{array}$ & 7 & $1.417,9$ & 226,0 & $1.145,0$ & $1.300,0$ & $1.350,0$ & $1.597,5$ & $1.832,5$ \\
\hline Salsicha & 32 & 943,9 & 286,4 & 344,0 & 700,0 & 880,0 & $1.110,0$ & $1.478,0$ \\
\hline Presuntaria & 45 & $1.329,9$ & 810,0 & 531,7 & 892,5 & $1.160,0$ & $1.560,0$ & $5.600,0$ \\
\hline
\end{tabular}

${ }^{a}$ Meta definida por porção do produto. Conteúdo de sódio $(\mathrm{mg})$ por porção: média $=936,3$; DP $=186,7 ; \mathrm{p} 25=912 ; \mathrm{p} 50=963$; p75 $=1020 .{ }^{b} \mathrm{mg}$ sódio/100mL do produto pronto para consumo. DP: desvio padrão; min: valor mínimo; p: percentil; máx: valor máximo.

Fonte: Autoras.

$\mathrm{mg} / 100 \mathrm{~g})$ ]. Entre as 32 categorias, $25,0 \%$ apresentaram conteúdo médio de sódio superior ao limite estabelecido pelos acordos voluntários de redução da substância ("mistura para bolo aerado", "mistura para bolo cremoso", "salgadinho de milho", "biscoito salgado", "requeijão", "mortadela conservada em refrigeração", "mortadela conservada em temperatura ambiente" e "presuntaria”). Comparando as medianas de sódio observadas às metas estabelecidas nos acordos, quatro categorias estavam acima do ponto de corte ("mistura para bolo aerado", "mistura para bolo cremoso", "linguiça frescal" e "mortadela conservada em refrigeração").

A Tabela 2 apresenta a proporção de alimentos adequados em relação aos limites propostos pelos acordos voluntários de redução de sódio e 
pelo modelo de perfil nutricional da OPAS para o conteúdo da substância. Considerando todos os produtos, $77,7 \%$ estavam adequados segundo os acordos voluntários de redução de sódio. As categorias com maior proporção de produtos dentro das metas dos acordos voluntários foram "bisnaguinha" (100,0\%), "tempero para arroz" (100,0\%), "cereal matinal" (97,5\%), "empanado" $(95,3 \%)$ e "maionese" (95,2\%). As categorias com menor proporção de produtos cumprindo os limites definidos pelos acordos foram "mortadela conservada em refrigeração" (23,8\%), "mistura

Tabela 2. Proporção de alimentos com conteúdo de sódio igual ou inferior aos limites estabelecidos pelos acordos voluntários de redução de sódio e pelo modelo de perfil nutricional da Organização Pan-Americana da Saúde, e concordância entre os dois critérios, segundo categoria de alimentos.

\begin{tabular}{|c|c|c|c|c|}
\hline Categoria de alimentos & $\mathbf{n}$ & $\begin{array}{c}\text { \% conformidade } \\
\text { com acordos } \\
\text { voluntários (IC } \\
95 \%) \\
\end{array}$ & $\begin{array}{l}\% \text { conformidade } \\
\text { com OPAS (IC 95\%) }\end{array}$ & $\mathrm{k}(\mathrm{IC}$ 95\%) \\
\hline Macarrão instantâneo & 80 & $96,3(88,9-98,8)$ & 0,0 & 0,000 \\
\hline Pão de forma & 112 & $93,8(87,4-97,0)$ & $13,4(8,2-21,1)$ & $0,020(0,002-0,038)$ \\
\hline Bisnaguinha & 9 & 100,0 & $33,3(10,3-68,5)$ & 0,000 \\
\hline Bolo pronto sem recheio & 90 & $78,9(69,2-86,1)$ & $73,3(63,2-81,5)$ & $0,726(0,560-0,893)$ \\
\hline Bolo pronto recheado & 46 & $91,3(78,8-96,7)$ & $91,3(78,8-96,7)$ & $0,726(0,365-1,000)$ \\
\hline Mistura para bolo aerado & 78 & $29,5(20,4-40,6)$ & $38,5(28,3-49,7)$ & $0,745(0,592-0,898)$ \\
\hline Mistura para bolo cremoso & 8 & $37,5(11,5-73,5)$ & 100,0 & 0,000 \\
\hline Salgadinho de milho & 48 & $60,4(46,0-73,3)$ & $10,4(4,4-22,9)$ & $0,142(0,017-0,266)$ \\
\hline Batata frita/palha & 81 & $80,2(70,1-87,6)$ & $80,2(70,1-87,6)$ & $0,922(0,816-1,000)$ \\
\hline Maionese & 21 & $95,2(71,8-99,4)$ & $4,8(0,6-28,2)$ & $0,005(-0,009-0,019)$ \\
\hline Biscoito doce & 37 & $89,2(74,2-95,9)$ & $94,6(80,5-98,7)$ & $0,641(0,189-1,000)$ \\
\hline Biscoito salgado & 45 & $64,4(49,4-77,1)$ & $6,7(2,1-18,9)$ & $0,076(-0,012-0,164)$ \\
\hline Biscoito recheado & 103 & $84,5(76,1-90,3)$ & $99,0(93,4-99,9)$ & $0,101(-0,083-0,286)$ \\
\hline Cereal matinal & 157 & $97,5(93,4-99,0)$ & $92,4(87,0-95,6)$ & $0,350(0,048-0,652)$ \\
\hline Margarina & 14 & $85,7(55,9-96,6)$ & $50,0(25,2-74,8)$ & $0,286(-0,066-0,637)$ \\
\hline Caldo pó/cubo & 38 & $78,9(63,0-89,2)$ & $2,6(0,4-16,8)$ & $0,014(-0,015-0,044)$ \\
\hline Tempero em pasta & 31 & $93,5(77,1-98,4)$ & $16,1(6,8-33,7)$ & $0,026(-0,016-0,068)$ \\
\hline Tempero para arroz & 14 & 100,0 & 0,0 & 0,000 \\
\hline Demais temperos & 66 & $89,4(79,3-94,9)$ & 0,0 & 0,000 \\
\hline Queijo muçarela & 53 & $66,0(52,3-77,5)$ & $18,9(10,4-31,8)$ & $0,214(0,075-0,352)$ \\
\hline Requeijão & 85 & $55,3(44,6-65,5)$ & $1,2(0,2-8,0)$ & $0,019(-0,018-0,056)$ \\
\hline Sopa & 43 & $90,7(77,5-96,5)$ & $2,3(0,3-15,1)$ & $0,005(-0,006-0,016)$ \\
\hline Sopa individual/ instantânea & 12 & $75,0(43,4-92,2)$ & 0,0 & 0,000 \\
\hline Empanado & 43 & $95,3(83,0-98,9)$ & 0,0 & 0,000 \\
\hline Hambúrguer & 39 & $79,5(63,8-89,5)$ & $25,6(14,3-41,7)$ & $0,163(0,030-0,297)$ \\
\hline $\begin{array}{l}\text { Linguiça cozida conservada em } \\
\text { refrigeração }\end{array}$ & 22 & $81,8(59,7-93,2)$ & $4,5(0,6-27,1)$ & $0,021(-0,024-0,066)$ \\
\hline Linguiça frescal & 13 & $61,5(33,3-83,7)$ & 0,0 & 0,000 \\
\hline $\begin{array}{l}\text { Mortadela conservada em } \\
\text { refrigeração }\end{array}$ & 60 & $45,0(32,9-57,8)$ & 0,0 & 0,000 \\
\hline $\begin{array}{l}\text { Mortadela conservada em } \\
\text { temperatura ambiente }\end{array}$ & 21 & $23,8(10,0-46,7)$ & $4,8(0,6-28,2)$ & $0,276(-0,164-0,716)$ \\
\hline $\begin{array}{l}\text { Linguiça cozida conservada em } \\
\text { refrigeração }\end{array}$ & 7 & $71,4(29,8-93,6)$ & 0,0 & 0,000 \\
\hline Salsicha & 32 & $81,3(63,7-91,4)$ & 0,0 & 0,000 \\
\hline Presuntaria & 45 & $55,6(40,8-69,4)$ & 0,0 & 0,000 \\
\hline Total & 1.553 & $77,7(75,5-79,7)$ & $35,9(33,5-38,3)$ & $0,199(0,169-0,230)$ \\
\hline
\end{tabular}

IC: intervalo de confiança; k: coeficiente de kappa de Cohen. 
para bolo aerado" (29,5\%), "mistura para bolo cremoso" (37,5\%) e "linguiça frescal" (45,0\%). Os produtos com conteúdo de sódio acima das metas pertenciam a mais de cem diferentes marcas. Dezessete marcas continham ao menos cinco produtos em desconformidade e juntas foram responsáveis por $52,7 \%$ desses itens (183 de 347). Sete dessas marcas pertenciam a empresas vinculadas a associações de alimentos que firmaram o acordo. Entre as dez restantes, quatro eram marcas próprias de supermercados.

Quando analisados em relação ao limite proposto pela OPAS, apenas $35,9 \%$ dos alimentos estavam adequados para a quantidade de sódio. As categorias com maior proporção de alimentos dentro do limite estipulado pela OPAS foram "mistura para bolo cremoso" (100,0\%), "biscoito recheado" (99,0\%), "biscoito doce" (94,6\%), "cereal matinal" $(92,4 \%)$ e "bolo pronto recheado" $(91,3 \%)$. Por outro lado, em cerca de um terço das categorias $(31,3 \%)$, nenhum produto apresentou conteúdo de sódio igual ou abaixo ao limite proposto pelo modelo de perfil nutricional da OPAS, e em outras 16 (50,0\%) a proporção de alimentos com conteúdo adequado de sódio foi igual ou inferior a $50 \%$.

De forma geral, considerando todos os produtos avaliados, a concordância entre os dois critérios foi fraca, com valor de kappa igual a 0,199. Apenas cinco categorias apresentaram concordância substancial ou superior $(k>0,60)$ : "batata frita/palha", "mistura para bolo aerado", "bolo pronto sem recheio", "bolo pronto recheado" e "biscoito doce". A grande maioria das categorias (23 de 32, ou 71,9\%) apresentou concordância fraca ou pobre.

\section{Discussão}

Nossos achados mostraram que cerca de um quarto dos produtos comercializados nas maiores redes de supermercado do Brasil e pertencentes às categorias selecionadas para redução voluntária de sódio ainda não atingiam as metas estabelecidas nos acordos de cooperação em 2017. Uma parte ainda maior dos itens avaliados $(64,1 \%)$ apresentou conteúdo de sódio superior ao limite proposto no modelo de perfil nutricional da OPAS. Os dois critérios apresentaram fraca concordância para classificar produtos com alto conteúdo de sódio, ainda que ambos visem à prevenção de DCNT.

Estudos recentes sobre o tema apontaram uma redução progressiva na quantidade de só- dio em alimentos comercializados no Brasil e, no geral, alta proporção de produtos cumprindo as metas dos acordos voluntários. Nilson e colaboradores avaliaram 20 categorias incluídas nos primeiros acordos voluntários e encontraram uma redução significativa, que variou de $8 \%$ a $34 \%$, no conteúdo médio de sódio nos alimentos de 13 categorias entre 2011 e $2017^{21}$. Outro estudo que incluiu as mesmas categorias de alimentos mostrou que, entre 2011 e 2013, em todas as categorias de alimentos estudadas, mais de $85 \%$ dos produtos alcançaram a meta de sódio estabelecida nos acordos voluntários até aquele momento ${ }^{22}$.

Algumas diferenças metodológicas podem ter contribuído para que nossos resultados tenham sido diferentes dos anteriormente mencionados. Incluímos na análise produtos de todas as categorias contempladas nos acordos e as metas definidas até o ano de 2017. Algumas das categorias com menor proporção de produtos cumprindo as metas, como linguiças, mortadelas e presuntaria, foram apenas mais recentemente incluídas nos acordos e não foram avaliadas por Nilson e colaboradores, que estudaram as categorias de alimentos incluídas nos acordos de $2011^{22}$. Entretanto, quando comparadas algumas das mesmas categorias, encontramos menor adequação do que a relatada por Nilson e colaboradores-para "bolo pronto sem recheio", "mistura para bolo aerado", "mistura para bolo cremoso", "salgadinhos de milho" e "biscoitos salgados", por exemplo. Essa diferença pode ser ao menos em parte explicada pelo fato de termos incluído todos os produtos encontrados nos supermercados durante a coleta, e não apenas aqueles fabricados por empresas vinculadas à $\mathrm{ABIA}^{22}$. Nossos resultados mostraram que a maioria das marcas que mais contribuíram com itens em desacordo com as metas não eram de empresas vinculadas às associações de alimentos signatárias. Assim, apesar de esperado que os acordos desencadeassem mudanças no mercado também entre empresas que não os assinaram, nosso estudo sugere que o efeito neste último grupo ficou aquém daquele encontrado para as empresas vinculadas à ABIA. Portanto, as mudanças no mercado de alimentos podem não ter sido tão acentuadas quanto aquelas anteriormente mostradas. O Brasil, por exemplo, apresentou a menor proporção de cumprimento das metas regionais entre 14 países da América Latina e do Caribe ${ }^{23}$.

Nossa comparação entre os acordos voluntários e o modelo de perfil nutricional da OPAS indicou que os dois métodos são discordantes ao classificar um produto como com conteúdo 
de sódio compatível com a prevenção de DCNT. As metas dos acordos voluntários foram baseadas nas médias encontradas por categorias, excluindo os valores extremos, de modo que, de partida, cerca de metade dos produtos já estavam adequados. Em resumo, as metas brasileiras foram determinadas de forma a atingir sobretudo os produtos com grandes quantidades de sódio disponíveis no mercado, não sendo capazes de assegurar que os produtos das diversas categorias terão um perfil nutricional adequado ${ }^{24}$. $\mathrm{O}$ acordo voluntário de redução de sódio praticado no Reino Unido, por exemplo, inclui um número maior de categorias de alimentos (cerca de 80) e as metas, em geral, são mais exigentes, principalmente para produtos cárneos como linguiças e hambúrgueres ${ }^{25}$. Algumas categorias incluídas no Reino Unido, como pizzas e sanduíches, também apresentam altas densidades de sódio no Brasili ${ }^{26}$, porém tais alimentos ainda não são contemplados pelos acordos. A deficiência no monitoramento também é um fator que limita a ação dos acordos voluntários na prevenção e no controle das DCNT. Estudo que avaliou os informes técnicos publicados pela Agência Nacional de Vigilância Sanitária (Anvisa), responsável pelo monitoramento das metas de redução junto ao MS, mostrou que faltava padronização quanto a categorias de alimentos, número de amostras e regiões do país incluídas no processo ${ }^{27}$.

Diversos países já adotaram medidas com o objetivo de reduzir o consumo de sódio na população ${ }^{28}$, no entanto, a estimativa é que este consumo ainda seja superior ao nível recomendado para prevenção de DCNT em todos os países e regiões do mundo ${ }^{29}$. Algumas nações, como Reino Unido e Finlândia, são exemplos de sucesso de acordos voluntários com a indústria de alimentos e conseguiram, ao longo das últimas décadas, a redução no conteúdo de sódio em produtos, no consumo populacional e nos níveis de pressão arterial e doenças associadas ${ }^{30-32}$. Apesar do progresso registrado na Finlândia, que reduziu o consumo populacional de 12 gramas para cerca de 9 gramas por dia entre 1979 e 2002, Laatikainen e colaboradores estimaram que ainda seriam necessários 35 e 26 anos, respectivamente, para que homens e mulheres do país chegassem a cinco gramas diários de consumo de sódio caso mantida a tendência de redução observada no período $^{31}$. Mais recentemente, Argentina e África do Sul adotaram medidas legislativas para redução de sódio em diversas categorias de alimentos. As vantagens apontadas sobre a reformulação obrigatória incluem a possibilidade de sanções fiscais, a abrangência a todos os fabricantes e a maior estabilidade da medida em diferentes governos $^{28}$. As críticas às reformulações voltadas para nutrientes críticos, como é o caso do sódio, são de que a estratégia tem limitações importantes, entre elas a incapacidade de fazer com que os produtos ultraprocessados reformulados tenham adequado perfil nutricional e sua racionalidade em mudar o consumo de nutrientes sem mudar o padrão alimentar, o que pode legitimar o consumo de tais produtos ${ }^{33}$.

Além da reformulação, outras estratégias podem ser implementadas para reduzir o consumo de sódio da população. Entre elas estão a tributação e a rotulagem nutricional adequada, incluindo a presença de advertências frontais indicando nutrientes críticos em excesso. O México adotou, em 2014, um imposto sobre bebidas açucaradas e alimentos não essenciais com alta densidade energética, como salgadinhos, doces e cereais matinais, e um ano após a implementação da medida foi observada menor compra dos produtos regulados ${ }^{34}$. Na Hungria, a tributação de produtos não saudáveis também foi efetiva na redução do consumo de categorias de alimentos como salgadinhos e na escolha de opções mais saudáveis, tanto pelo preço quanto pelo maior conhecimento sobre os produtos ${ }^{35}$. O uso de advertências frontais, por sua vez, é mencionado no documento "Salt-smart Americas: a guide for country-level action" como uma medida complementar à reformulação de alimentos, com o objetivo de diminuir o consumo de sódio da população $0^{6}$. Na América Latina, Chile, Uruguai, Peru e México já adotaram selos de advertência frontal nas embalagens de alimentos. Estudos realizados no Brasil demonstraram que a presença de alertas apontando o alto conteúdo de nutrientes críticos no rótulo de alimentos podem auxiliar consumidores a fazerem escolhas alimentares mais saudáveis ${ }^{36,37}$.

O presente estudo se destaca pelo tamanho e abrangência de sua amostra de alimentos embalados comercializados no Brasil. No entanto, algumas limitações devem ser consideradas. Para algumas categorias de alimentos, o número de produtos encontrado foi pequeno e não se pode descartar a ocorrência de perdas de alguns alimentos na coleta de dados. Contudo, os artigos publicados previamente também encontraram número pequeno de alimentos em algumas categorias $^{21,22}$, indicando que a variedade de produtos de alguns segmentos pode ser restrita. Também é uma limitação o fato de não termos incluído outros tipos de estabelecimentos de venda de ali- 
mentos. Contudo, os supermercados são o principal local de compras de alimentos no Brasil e fornecem cerca de $60 \%$ das calorias adquiridas para consumo domiciliar ${ }^{38}$. Além disso, as informações utilizadas foram aquelas disponibilizadas pelas empresas nos rótulos dos alimentos, sem a realização de análises laboratoriais para verificar o conteúdo reportado. Nosso estudo também não considerou a participação de mercado dos produtos avaliados, portanto não foi capaz de identificar o conteúdo de sódio entre os produtos mais consumidos pelos brasileiros.

Considerando o consumo de sódio no país, que supera em mais de duas vezes o limite recomendado, e o crescente consumo de produtos ultraprocessados, são necessárias medidas regulatórias e mais rigorosas para que seja atingida a meta de redução do consumo de sódio na população brasileira. O prazo para que os alimentos embalados alcancem os limites de sódio acordados ter- mina em 2020 e a tendência é que o Brasil ainda se mantenha distante do resultado desejado quanto ao consumo populacional desse nutriente. Recomendamos a ampliação das medidas a um maior número de categorias de produtos disponíveis no mercado, em especial para ultraprocesados, como pizzas e sanduiches, e o aprimoramento das metas para que sejam mais rígidas, como em outros países, e, idealmente, mais próximas do nível proposto pela OPAS para prevenção de DCNT. Por fim, é importante salientar as limitações inerentes aos acordos voluntários, quando comparados a medidas regulatórias mandatórias que deveriam ser priorizadas. Outras medidas como a inclusão de advertências nos rótulos indicando o alto conteúdo de sódio e ações educativas de conscientização da população sobre o uso e consumo de sal devem ser concomitantemente implementadas a fim de obter redução no nível de consumo de sódio populacional.

\section{Colaboradores}

CZ Ricardo e BC Salvador propuseram o estudo. CZ Ricardo conduziu a análise dos dados, escreveu a versão preliminar, revisou, editou e aprovou a versão final. GC Andrade e BC Salvador contribuíram com a escrita da versão preliminar do artigo, revisaram, editaram e aprovaram a versão final. LA Mais revisou, editou e aprovou a versão final. AC Duran e APB Martins são as coordenadoras do projeto e revisaram, editaram e aprovaram a versão final enviada para submissão.

\section{Referências}

1. World Health Organization (WHO). Noncommunicable diseases country profiles 2018. Geneva: WHO; 2018.

2. Strazzullo P, D'Elia L, Kandala NB, Cappuccio FP. Salt intake, stroke, and cardiovascular disease: meta-analysis of prospective studies. BMJ 2009; 339:b4567.

3. He FJ, Tan M, Ma Y, MacGregor GA. Salt reduction to prevent hypertension and cardiovascular disease: JACC state-of-the-art review. J Am Coll Cardiol 2020; 75(6):632-647.

4. Webb M, Fahimi S, Singh GM, Khatibzadeh S, Micha R, Powles J, Mozaffarian D. Cost effectiveness of a government supported policy strategy to decrease sodium intake: global analysis across 183 nations. BMJ 2017; 356:i6699.

5. World Health Organization (WHO). Global action plan for the prevention and control of noncommunicable diseases 2013-2020. Geneva: WHO; 2013.

6. Pan American Health Organization (PAHO). Salt-smart Americas: a guide for country-level action. Washington, DC: PAHO; 2013.

7. Mill JG, Malta DC, Machado ÍE, Pate A, Pereira CA, Jaime PC, Szwarcwald CL, Rosenfeld LG. Estimation of salt intake in the Brazilian population: results from the 2013 National Health Survey. Rev Bras Epidemiol 2019; 22(Suppl. 2):E190009.SUPL.2.

8. Sarno F, Claro RM, Levy RB, Bandoni DH, Monteiro CA. [Estimated sodium intake for the Brazilian population, 2008-2009]. Rev Saude Publica 2013; 47(3):571-578. 
9. Moura Souza A, Bezerra IN, Pereira RA, Peterson KE, Sichieri R. Dietary sources of sodium intake in Brazil in 2008-2009. Journal of the Academy of Nutrition and Dietetics 2013; 113(10):1359-1365.

10. Nilson EAF, Jaime PC, Resende DO. Iniciativas desenvolvidas no Brasil para a redução do teor de sódio em alimentos processados. Rev Panam Salud Publica 2012; 32:6.

11. Brasil. Termo de Compromisso 004/2011 de 7 de abril de 2011. Estabelece as metas nacionais para a redução do teor de sódio em alimentos processados no Brasil. Brasília: Diário Oficial da União; 2011.p. 81.

12. Brasil. Termo de Compromisso 035/2011 de 13 de dezembro de 2011. Estabelece as metas nacionais para a redução do teor de sódio em alimentos processados no Brasil. Brasília: Diário Oficial da União; 2011.

13. Brasil. Termo de Compromisso de 5 de novembro de 2013. Estabelece as metas nacionais para a redução do teor de sódio em alimentos processados no Brasil. Brasília: Diário Oficial da União; 2013.

14. Brasil. Termo de Compromisso de 28 de agosto de 2012. Estabelece as metas nacionais para a redução do teor de sódio em alimentos processados no Brasil. Brasília: Diário Oficial da União; 2012. p. 124.

15. Brasil. Termo de Compromisso 005/2017 de 13 de junho de 2017. Estabelece as metas nacionais para a redução do teor de sódio em alimentos processados no Brasil. Brasília: Diário Oficial da União; 2017.

16. Euromonitor International. Grocery retailers in Brazil. 2016.

17. Instituto Brasileiro de Geografia e Estatística (IBGE). Censo demográfico 2010. Rio de Janeiro: IBGE; 2010.

18. Kanter R, Reyes M, Corvalán C. Photographic methods for measuring packaged food and beverage products in supermarkets. Curr Dev Nutr 2017; 1(10):e001016.

19. Pan American Health Organization (PAHO). Pan American Health Organization Nutrient Profile Model. Washington, DC: PAHO; 2016.

20. Watson PF, Petrie A. Method agreement analysis: a review of correct methodology. Theriogenology 2010; 73(9):1167-1179.

21. Nilson EAF, Spaniol AM, Gonçalves VSS, Moura I, Silva SA, L'Abbé M, Jaime PC. Sodium reduction in processed foods in Brazil: analysis of food categories and voluntary targets from 2011 to 2017. Nutrients 2017; 9(7):742.

22. Nilson EAF, Spaniol AM, Gonçalves VSS, Oliveira ML, Campbell N, L'Abbé M, Jaime PC. The impact of voluntary targets on the sodium content of processed foods in Brazil, 2011-2013. J Clin Hypertens (Greenwich) 2017; 19(10):939-945.

23. Arcand J, Blanco-Metzler A, Benavides Aguilar K, L'Abbe MR, Legetic B. Sodium levels in packaged foods sold in 14 Latin American and Caribbean countries: a food label analysis. Nutrients 2019; 11(2):369.

24. Martins APB. Redução de sódio em alimentos: uma análise dos acordos voluntários no Brasil. São Paulo: Instituto Brasileiro de Defesa do Consumidor; 2014.

25. Public Health England (PHE). Salt reduction targets for 2017. London: PHE; 2017.

26. Souza AM, Bezerra IN, Pereira RA, Peterson KE, Sichieri R. Dietary sources of sodium intake in Brazil in 2008-2009. J Acad Nutr Diet 2013; 113(10):1359-1365.
27. Martins APB, Andrade GC, Bandoni DH. Avaliação do monitoramento do teor de sódio em alimentos: uma análise comparativa com as metas de redução voluntárias no Brasil. Vigil Sanit Debate 2015; 3:9.

28. Webster J, Trieu K, Dunford E, Hawkes C. Target salt 2025: a global overview of national programs to encourage the food industry to reduce salt in foods. $\mathrm{Nu}$ trients 2014; 6(8):3274-3287.

29. Powles J, Fahimi S, Micha R, Khatibzadeh S, Shi P, Ezzati M, Engell RE, Lim SS, Danaei G, Mozaffarian D; Global Burden of Diseases Nutrition and Chronic Diseases Expert Group (NutriCoDE). Global, regional and national sodium intakes in 1990 and 2010: a systematic analysis of $24 \mathrm{~h}$ urinary sodium excretion and dietary surveys worldwide. BMJ Open 2013; 3(12): $\mathrm{e} 003733$.

30. He FJ, MacGregor GA. A comprehensive review on salt and health and current experience of worldwide salt reduction programmes. J Hum Hypertens 2009; 23(6):363-384.

31. Laatikainen T, Pietinen P, Valsta L, Sundvall J, Reinivuo H, Tuomilehto J. Sodium in the Finnish diet: 20year trends in urinary sodium excretion among the adult population. Eur J Clin Nutr 2006; 60(8):965-790.

32. Laverty AA, Kypridemos C, Seferidi P, Vamos EP, Pearson-Stuttard J, Collins B, Capewell S, Mwatsama M, Cairney P, Fleming K, O’Flaherty M, Millett C. Quantifying the impact of the Public Health Responsibility Deal on salt intake, cardiovascular disease and gastric cancer burdens: interrupted time series and microsimulation study. J Epidemiol Community Health 2019; 73(9):881-887.

33. Scrinis G, Monteiro CA. Ultra-processed foods and the limits of product reformulation. Public Health Nutr 2018; 21(1):247-252.

34. Batis C, Rivera JA, Popkin BM, Taillie LS. First-Year evaluation of Mexico's tax on nonessential energydense foods: an observational study. PLoS Med 2016; 13(7):e1002057.

35. World Health Organization (WHO). Assessment of the impact of a public health product tax. Budapest: WHO; 2015.

36. Sato PM, Mais LA, Khandpur N, Ulian MD, Bortoletto Martins AP, Garcia MT, Spinillo CG, Urquizar Rojas CF, Jaime PC, Scagliusi FB. Consumers' opinions on warning labels on food packages: A qualitative study in Brazil. Plos One 2019; 14(6):e0218813.

37. Khandpur N, Sato PM, Mais LA, Bortoletto Martins AP, Spinillo CG, Garcia MT, Urquizar Rojas CF, Jaime PC. Are front-of-package warning labels more effective at communicating nutrition information than traffic-light labels? A randomized controlled experiment in a Brazilian sample. Nutrients 2018; 10(6):688.

38. Machado PP, Claro RM, Canella DS, Sarti FM, Levy RB. Price and convenience: The influence of supermarkets on consumption of ultra-processed foods and beverages in Brazil. Appetite 2017; 116:381-388.

Artigo apresentado em 07/08/2020

Aprovado em 15/01/2021

Versão final apresentada em 17/01/2021

Editores-chefes: Romeu Gomes, Antônio Augusto Moura da Silva 